\title{
Uma Proposta de Ferramenta para Execução Descentralizada de Processos de Software
}

\author{
Adailton Magalhães Lima, Rodrigo Quites Reis \\ Laboratório de Engenharia de Software (LABES) \\ Programa de Pós-Graduação em Ciência da Computação \\ Universidade Federal do Pará (UFPA) \\ Belém, Pará, Brasil \\ \{adailton,quites\}@webapsee.com
}

\begin{abstract}
Communication and Coordination constitute major challenges for decentralized software development projects nowadays. These problems are often related to the organizational aspects of the involved parts - customers and suppliers. Therefore, this paper describes a proposal to provide support for decentralized process enactment in an open source Process-centered Software Engineering Environment, matching its functionalities with the requirements from process maturity models. Finally, the paper discusses some future directions of the research of this theme.

Resumo: Em projetos de desenvolvimento descentralizado de software existem problemas de comunicação e coordenação do processo de desenvolvimento, que frequentemente estão relacionadas com questões organizacionais dos envolvidos (clientes e fornecedores). Este trabalho descreve uma proposta para permitir a execução descentralizada de processos de software em um Sistema de Gestão baseado em Software Livre, relacionando as funcionalidades da proposta com os requisitos exigidos por modelos de maturidades de processos e por problemas citados pela literatura. Por fim, o texto aponta direções para pesquisa futura no tema.
\end{abstract}

\section{Introdução}

A Engenharia de Software evolui sistematicamente como área de pesquisa científica e prática na indústria através da incorporação de métodos, técnicas e ferramentas que contribuam para o aumento da qualidade do software produzido. Segundo [Fuggetta 2000a], a qualidade do software produzido é fortemente dependente da qualidade do processo usado no seu desenvolvimento. Ainda segundo [Fuggetta 2000a], o processo de desenvolvimento de software é formado por um conjunto coerente de políticas, estruturas organizacionais, tecnologias, procedimentos e artefatos necessários para conceber, desenvolver e manter um produto de software.

Atualmente a área de Tecnologia de Processos de Software vem ganhando destaque. Esta área envolve a construção de ambientes e ferramentas que atuam na modelagem, execução, simulação e evolução de processos de desenvolvimento de software. Segundo [Fuggetta 2000a], Ambientes de Desenvolvimento de Software que permitem a modelagem e execução de processos de software são genericamente denominados como PSEE (Process-Centered Software Engineering Environment). 
Segundo [Gimenes 1994], também são conhecidos como Ambientes de Desenvolvimento de Software Centrados em Processos (em português).

Segundo [Derniame 2002] a execução de modelos de processos de software é apoiada atualmente por vários PSEEs encontrados na literatura. Porém, o auxílio para a coordenação de atividades de forma descentralizada entre diversos PSEEs é uma extensão que somente alguns possuem. A maioria provê uma solução centralizada, baseada na arquitetura cliente-servidor [Lima 2006].

A coordenação descentralizada de atividades em projetos de desenvolvimento de software é uma necessidade corrente na comunidade de Engenharia de Software. Segundo [Moitra 2001], a descentralização de projetos de software entre diversas localidades distribuídas no globo é uma tendência atual do mercado de desenvolvimento de software. Dependendo da relação de negócio entre as partes envolvidas, pode-se caracterizar tais relações como Insourcing (relação de uma mesma empresa e suas filias) ou Outsourcing (relação de empresas diferentes) [Robinson 2004].

Neste contexto, este trabalho descreve a proposta de uma extensão a um PSEE existente para permitir a coordenação descentralizada de processos de software. Inicialmente são apresentados os conceitos relacionados com Desenvolvimento Descentralizado de Software (seção 2). Em seguida é apresentada uma visão geral sobre Ambientes de Desenvolvimento de Software Centrados em Processos (seção 3), com a apresentação das principais características do ambiente WebAPSEE (seção 4). A partir dos principais aspectos envolvidos na proposta deste trabalho e das propostas encontradas na literatura, são apresentados os aspectos da extensão para descentralização de processos ao ambiente WebAPSEE, onde também é discutida a correspondência da proposta com a melhoria da qualidade de processos de software (seção 5). Por fim a seção 6 apresenta as conclusões e trabalhos futuros.

\section{Desenvolvimento Descentralizado de Software}

Desenvolvimento Descentralizado de Software é um campo de estudo profundamente influenciado pelo crescimento da demanda da indústria. Tecnologias de Software influenciam decisivamente a escala de projetos descentralizados de software. Do ponto de vista do cliente, é importante prover auxílio à monitoração dos processos adotados pelos fornecedores no sentido de prover uma avaliação das atividades subcontratadas. Do outro lado, a monitoração em tempo real em intervalos é útil para distinguir organizações desenvolvedoras de software (fornecedores) como um fator de qualidade [Ebert 2006].

O estado da prática atual neste campo de estudo é comumente baseado nas facilidades de repositórios compartilhados que são utilizados para monitoramento dos artefatos entregues e/ou modificados. No entanto, isto fornece apenas uma visão limitada dos fornecedores, já que não existe informação disponível a respeito do verdadeiro modelo de processos em execução. Por exemplo, poucas informações a respeito da realização das atividades "guarda-chuva" (que não possuam artefatos de saída definidos, como por exemplo, as revisões para gerência de qualidade) podem ser extraídas dos repositórios de artefatos. 


\subsection{Desafios para Desenvolvimento Descentralizado de Software}

Além das dificuldades inerentes ao desenvolvimento de software, projetos que são descentralizados geograficamente, não importando a distância geográfica entre os participantes, sofrem de problemas comuns, relacionados diretamente com os fatores de comunicação, gerência de dependência entre tarefas e artefatos, e a imediata notificação a respeito dos agentes e suas tarefas. As subseções a seguir irão descrever os principais problemas citados na literatura estudada a respeito deste tema.

\subsubsection{Comunicação}

Segundo [Herbsleb 2001], distância e comunicação são fatores inversamente proporcionais no desenvolvimento de software. Tanto fatores relacionados com o gerenciamento da interdependência de artefatos, quanto fatores relacionados com overhead de comunicação remota (realizada através de ligações interurbanas, e-mail, vídeo conferências) causam atrasos no atendimento dos objetivos do projeto e adicionam custos ao projeto final. Estas dificuldades se agravam quando as organizações envolvidas não possuem um nível de maturidade aceitável para o gerenciamento destas atividades [Ebert 2006].

Segundo [Moitra 2001], particularmente o início do desenvolvimento de software exige muita comunicação entre os envolvidos. Segundo [Ebert 2006], em fase inicial projetos de Outsourcing demandam cerca de $20 \%$ de esforço adicional devido aos problemas de comunicação e gerência.

Atividades de atualização e divulgação do estado do projeto são peças chave na manutenção do feedback entre as equipes envolvidas no projeto. A falha e/ou atraso na obtenção e/ou divulgação destas informações pode causar confusões e perdas de tempo no andamento do projeto [Sengupta 2006] [Ebert 2006]. Quando líderes de projeto disseminam informações inadequadas, suas equipes de desenvolvimento enfrentam dificuldades em definir quais as principais atividades do fluxo de execução do processo não têm seus estados conhecidos por todos, o que diminui as chances de melhor avaliação dos riscos do projeto [Ebert 2006].

\subsubsection{Gerência e Coordenação de Projetos}

Geralmente relacionadas a aspectos de gerência de projeto e processos de software, as atividades de determinação de papéis e responsabilidades entre os envolvidos precisam ter uma linguagem comum a todos os envolvidos no projeto para divulgação de suas informações [Ebert 2006].

Sem mecanismos efetivos de compartilhamento de informação, os gerentes envolvidos em projetos de desenvolvimento descentralizado de software não podem desfrutar de todos os benefícios oferecidos por este modo de trabalho. Assim, valiosas oportunidades de reuso (tanto de processos de desenvolvimento quanto de artefatos produzidos) são perdidas, as quais poderiam reduzir custos e tempo [Moitra 2001] [Sengupta 2006].

Como exemplo dos problemas decorrentes da falta de mecanismos de compartilhamento de informações de processos em projetos descentralizados, existe o problema de definição do nível de granulosidade das atividades para o modelo de processo a ser seguido. Segundo [Ebert 2006], gerentes que estão distantes fisicamente das equipes que coordenam, muitas vezes procurando manter um maior controle sobre 
as atividades, definem tarefas com uma granulosidade muito fina (ou seja, são criadas muitas "pequenas" atividades). Esta abordagem implica na sobrecarga criada pelo constante preenchimento de documentação de controle, o que freqüentemente acaba atrapalhando o andamento do processo pelo excesso de burocracia. O inverso - isto é, a definição de atividades muito genéricas - também é prejudicial ao andamento do projeto, pois dificulta o controle gerencial sobre o andamento das atividades.

\subsubsection{Gerência de Contratos}

No gerenciamento de projetos descentralizados de desenvolvimento de software, [Ebert 2006] destaca também o problema do gerenciamento insuficiente de contratos. Este problema consiste na falta de controle sobre os aspectos de qualidades e prazos definidos em contratos de desenvolvimento com terceiros.

Definir e gerenciar as responsabilidades entre clientes e fornecedores ajuda a evitar o jogo de "apontar culpados" ao final do projeto, pois durante o mesmo todos estavam cientes dos prazos e avisados no momento de atrasos ocorridos [Herbsleb 2001] [Ebert 2006]. Da mesma forma, a manutenção de um feedback contínuo entre as atividades de fornecedores para clientes pode proporcionar auxílio na monitoração dos objetivos definidos entre as partes.

\subsection{Relação com Modelos de Maturidade de Processos}

Levando em consideração a relevância da definição de processos de software de uma organização [Weber 2006] [CMMI 2007], os autores deste texto sugerem que as soluções tecnológicas para apoiar a monitoração de projetos descentralizados devem levar em consideração tanto o aspecto da produção dos artefatos quanto à monitoração dos processos em si. Além disso, as soluções devem estar vinculadas com as questões contratuais, de forma que exista explicitamente um modelo que norteie quais informações serão passadas do contratante para o fornecedor e vice-versa.

A aplicação de tais soluções, do ponto vista do cliente o conhecimento a respeito da situação corrente de desenvolvimento no fornecedor faz com que os riscos sejam mais claros, a ponto de permitir tomada de decisões antes dos riscos tornarem o projeto inviável ou exigirem medidas drásticas. Do ponto de vista do fornecedor, a transparência de processos com os clientes pode representar uma vantagem competitiva no mercado. Todavia, isto pode representar um risco para os negócios da organização, já que algumas atividades e artefatos representam seus interesses e objetivos estratégicos [Wells 2001], sendo que este sigilo de algumas informações deve também ser considerado nas propostas que procurem atender esta monitoração remota.

Desta forma, o melhor gerenciamento e controle das atividades de comunicação com os terceiros envolvidos ajuda a contornar os problemas de gerência e comunicação citados anteriormente. Segundo [Feldmann 2001], a garantia da qualidade em casos de desenvolvimento descentralizado também exige apoio para correta definição (modelagem) e execução de modelos de processos de software. Ainda segundo [Feldmann 2001], pela falta de ferramentas especializadas, muitas vezes ferramentas não necessariamente projetadas para desenvolvimento descentralizado são utilizadas.

\subsubsection{CMMI}

A definição de processos para gerência de relacionamentos com terceiros é exigida nos principais modelos de qualidade e maturidade de processos existentes. No CMMI 
[CMMI, 2007] a área de processos "Gerência integrada com fornecedores", que descreve os objetivos que uma organização deve obter em seus processos no relacionamento com fornecedores externos.

A gerência integrada com fornecedores tem o foco na gerência da aquisição de produtos e serviços, ao invés de desenvolvê-los. Há o propósito de gerenciar de forma pró-ativa para verificar se os produtos estão satisfazendo os requisitos do projeto e gerenciar os fornecedores selecionados enquanto existe a relação cooperativa entre cliente e fornecedor. As duas metas definidas pelo CMMI para a gerência integrada com fornecedores são:

I. Análise e seleção de fonte de produtos, ou seja, seleção de fornecedores.

II. Coordenação do trabalho com fornecedores. Isto é alcançado a partir da monitoração e análise do processo de engenharia do fornecedor.

\subsubsection{MPS.BR}

No modelo MPS.BR [Weber 2006], o nível de maturidade F exige a definição do "Processo de Aquisição" em casos que a organização tenha relações com fornecedores externos. Neste processo a aquisição é monitorada de forma que as condições especificadas são atendidas, tais como: custo, cronograma e qualidade e, se necessário, ações corretivas são conduzidas.

O modelo MPS.BR possui o "Guia de Aquisição", que tem o objetivo de oferecer uma alternativa de processo de aquisição para organizações, considerando também que este é um importante elemento indutor de melhorias de processos de organizações fornecedoras.

Similarmente ao modelo proposto pelo CMMI, o modelo MPS.BR prevê etapas de acompanhamento dos produtos desenvolvidos por fornecedores. Em casos de desenvolvimento de software externo, a descentralização de processos se aplica na medida em que o fornecedor adote um processo para desenvolvimento da demanda requerida pelo cliente.

\section{Ambientes de desenvolvimento de Software Centrados em Processos}

Segundo [Fuggetta 2000a], assume-se que há uma relação direta entre a qualidade dos processos com a qualidade dos produtos de software. Os PSEEs relacionam-se com o problema de como sistemas computadorizados podem ser utilizados no desenvolvimento de software, ou seja, o uso de software para ajudar o gerenciamento da construção de software. Sua finalidade principal é atender a requisitos organizacionais para auxiliar na coordenação das atividades relacionadas com o desenvolvimento de software.

Especificamente com relação à execução de processos entende-se que as soluções desenvolvidas devem estar integradas com as técnicas de modelagem de processos. Assim, a execução e modelagem de processos são atividades que ocorrem freqüentemente de forma intercalada, pois se deve permitir a execução de processos parcialmente definidos [Lima Reis 2003], assim como a modificação de processos durante a execução. Esse requisito representa uma situação na qual o gerente pode iniciar a execução de um processo enquanto decide como vai ser realizada uma atividade futura. 
A grande maioria dos PSEEs apresenta uma arquitetura centralizada, onde os processos são executados em um único ponto e a distribuição dos dados e serviços é fornecida a partir de chamadas remotas de procedimentos. Como exemplo de abordagem centralizada pode-se citar os ambientes WebAPSEE [Lima 2006], Estação TABA [Montoni 2006], e EPOS [Conradi 1994]. Como características técnicas desvantajosas para a configuração centralizada podem-se citar: existe um ponto único de falha do sistema; a escalabilidade do sistema é limitada pelo poder de processamento da máquina servidora; o tempo de resposta final é limitado pelo grau de concorrência no servidor.

Além das desvantagens técnicas citadas, os PSEEs que adotam arquitetura centralizada também possuem desvantagens do ponto de organizacional de empresas. Se houver a necessidade de compartilhamento de informações a respeito dos processos de uma organização, o acesso aos dados aos interessados poderá abrir brechas para que informações sigilosas sobre os processos da organização sejam acessadas indevidamente, o que é totalmente desaprovado segundo [Wells 2001]. Mesmo que a implantação de políticas de segurança entre usuários de diferentes organizações permita a filtragem de informações, os bancos de dados que contêm informações da organização estarão em um repositório centralizado que muitas vezes está fora do domínio da organização (em algum serviço de hospedagem remoto). Neste caso, ainda há o risco de acesso indevido por terceiros aos dados sigilosos da organização.

Como exemplo da abordagem descentralizada de coordenação de processos pode-se citar os ambientes Endeavors [Taylor 1996], Oz [Beanshaul 1998], SerendiptyII [Grundy 1998], PIE [Fuggetta 2000b], Genesis [Ritrovato 2002] e SwinDeW [Yan 2003].

\section{Ambiente WebAPSEE}

WebAPSEE é um PSEE desenvolvido desde 2004 no contexto do Laboratório de Engenharia de Software ${ }^{1}$ da UFPA [Lima 2006], onde a versão atual é baseada nos modelos propostos por [Lima Reis 2003] e ainda está em desenvolvimento baseado principalmente nos modelos de [Reis 2002a, 2002b] e outros trabalhos de pesquisa ainda em andamento.

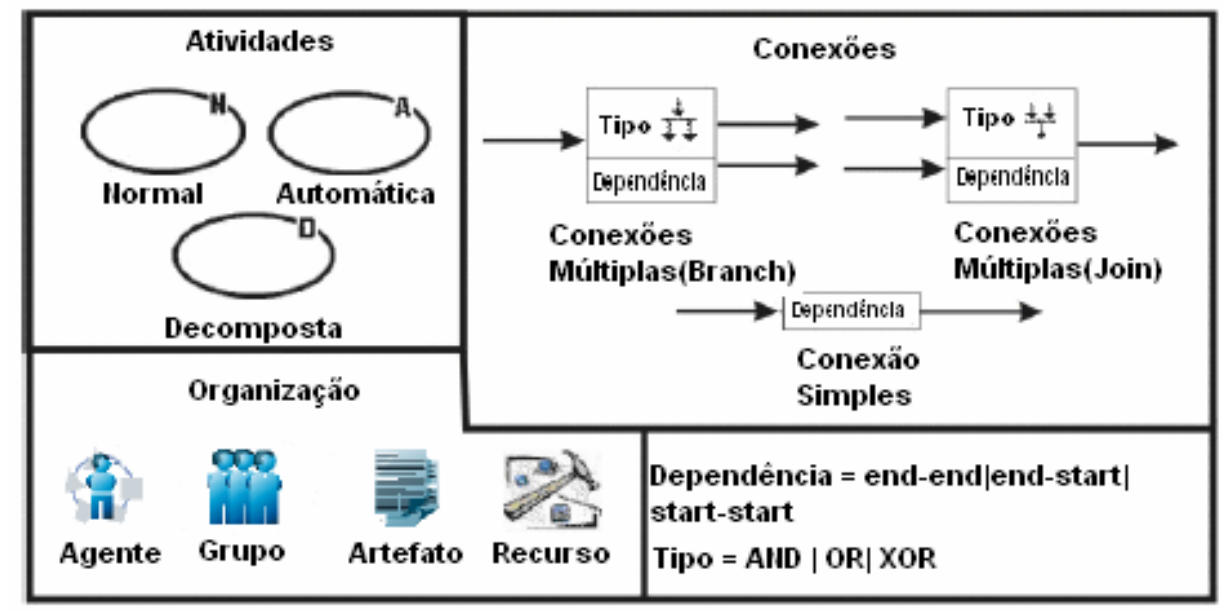

Figura 1 - Representação gráfica para as principais construções da Linguagem de Modelagem de Processo do WebAPSEE.

\footnotetext{
${ }^{1}$ Maiores informações disponíveis em http://www.labes.ufpa.br
} 


\subsection{A Linguagem Visual de Modelagem de Processo}

WebAPSEE-PML é a linguagem gráfica usada para modelar processos no ambiente WebAPSEE [Lima 2006] O meta-modelo do ambiente WebAPSEE inclui informação sobre os componentes da linguagem. Esta linguagem foi derivada da APSEE-PML original definida em [Lima Reis 2003]. Nesta abordagem, uma linguagem visual é definida por um alfabeto e uma gramática, onde as sintaxes abstrata e concreta são fornecidas.

Segundo [Lima Reis 2003], do ponto de vista da sincronização de eventos em processo-atividade, a execução de processo leva em consideração os seguintes elementos: as atividades e seus fragmentos (sub-atividades); a disponibilidade de recursos; e as pré e pós-condições das atividades.

A notação gráfica da WebAPSEE-PML é resumida pela Figura 1. Informações adicionais sobre atividades e conexões (isto é, métricas, estimativas, cargos, e scripts de atividades normais) são definidas através de formulários textuais específicos.

\subsection{Manager Console}

É utilizado um formalismo de modelagem para o WebAPSEE que permite representação gráfica de processos, tal como ilustrado na imagem localizada ao fundo da Figura 2, que mostra o componente denominado Manager Console. O ambiente WebAPSEE permite a integração de vários serviços de gerência de processos, incluindo modelagem, execução, visualização, instanciação e resposta a eventos da execução. Através do Manager Console, o Gerente do Processo de Desenvolvimento de software pode Modelar Processos, Gerenciar Execução de Processos, Visualizar Relatórios do Processo e Gerenciar informações da organização, como artefatos, pessoas e recursos, através de um Editor Gráfico. O Manager Console permite ainda o cadastro e gerência de projetos, recursos, artefatos, gerência de políticas.

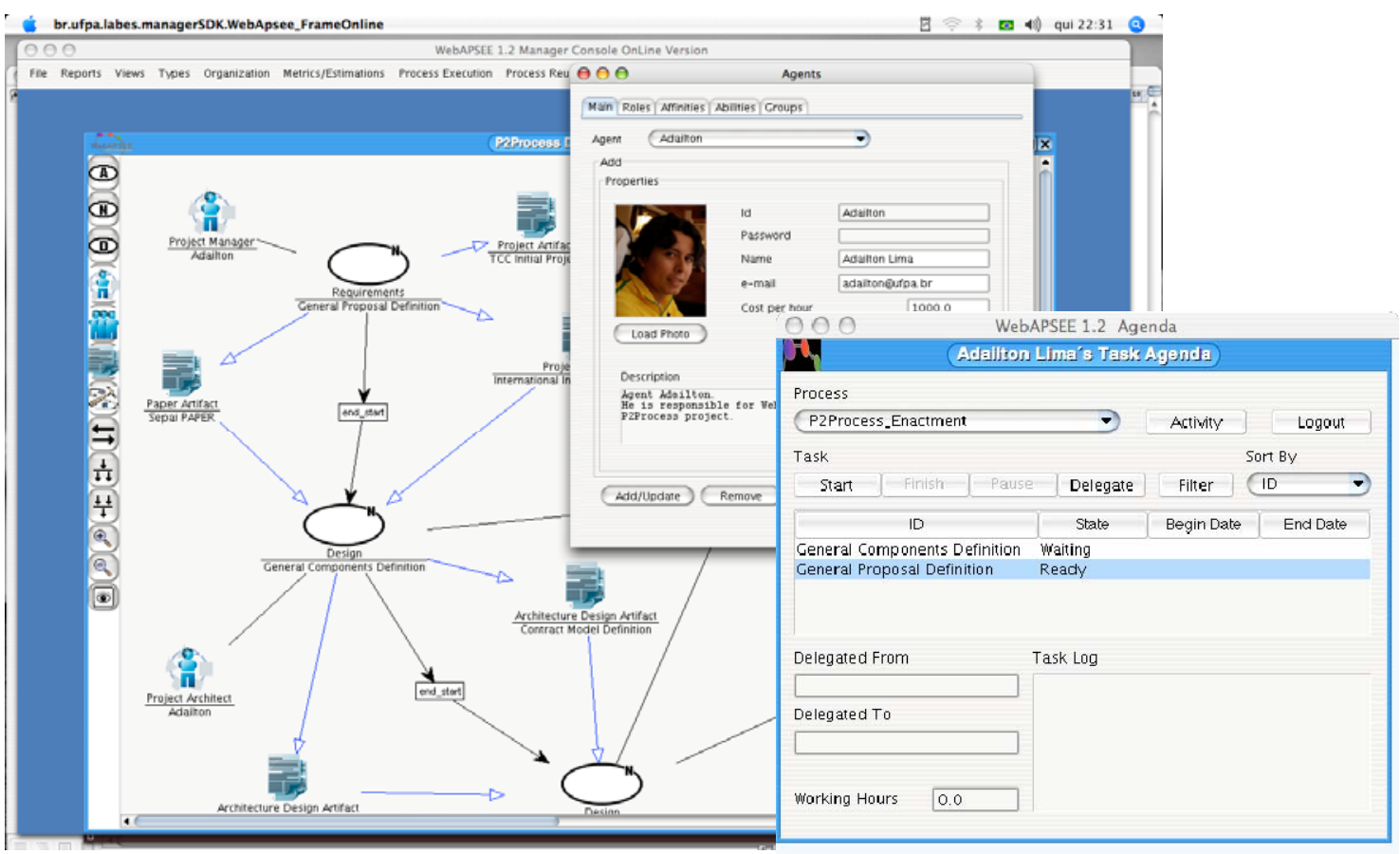

Figura 2 - Tela de uso do Ambiente WebAPSEE - Manager Console e Task Agenda 


\subsection{Task Agenda}

Através da Agenda (Task Agenda), o desenvolvedor visualiza os processos de software em execução nos quais está inserido como uma lista de tarefas a serem realizadas. Assim, o desenvolvedor interage com a agenda fornecendo feedback sobre o andamento dessas tarefas. A Figura 2 contém no canto inferior direito a ilustração de uma tela de uso da ferramenta Task Agenda. A Agenda representa a visão de um Agente a partir das tarefas que lhe são atribuídas para um determinado processo.

\section{Um Modelo de Execução Descentralizada para o Ambiente WebAPSEE}

A proposta deste trabalho está inserida no contexto do uso de ferramentas para auxiliar na gestão de projetos descentralizados de software. De maneira geral a proposta de "descentralizar a execução de processos" consiste em coordenar diversas atividades de um fluxo de atividades definido, sendo que a responsabilidade de coordenação de tais atividades pode estar distribuída entre diversas instâncias de um (ou mais) PSEE(s). Com isto, as instâncias do ambiente que participam da rede devem interagir para que seja mantida a consistência e completeza dos dados do ambiente e dos processos compartilhados.

A execução descentralizada de processos de software é apoiada por um bom número de PSEEs listados na literatura, os quais utilizam diferentes técnicas de distribuição. Encontram-se exemplos que fornecem distribuição de dados através de middleware tradicional [Ritrovato 2002], embora haja uma tendência atual em se identificar abordagens que utilizam tecnologias peer-to-peer [Yan 2003] [Grundy 1998].

\subsection{Solução Proposta}

De acordo com o escopo ilustrado na Figura 3, o componente denominado "Servidor" representa o site onde é modelado inicialmente o modelo de processos de software que posteriormente será descentralizado. A partir da definição da localização dos ambientes que são responsáveis pela execução de partes específicas do processo, entram em ações os nós identificados por "Nó A” e “Nó B”. Estes nós são entidades responsáveis pela coordenação das atividades que lhe foram atribuídas por outro host, além de suas atividades definidas localmente. As setas pontilhadas indicam a relação de cada site com seus clientes locais, assim como ocorre na versão atual do ambiente WebAPSEE. As setas espessas, com indicadores de direção nos dois sentidos, interligam os sites ilustrados na Figura 3 e representam os canais de comunicação para troca de informações entre os diversos sites ou peers da rede de instâncias de ambientes participantes da rede. Convém observar que o protótipo atual que está sendo desenvolvido se baseia em redes peer-to-peer.

Independentemente de haver ou não processos descentralizados definidos entre os hosts, a proposta é que os ambientes continuem funcionando como servidores locais às instâncias de Agendas e Manager Consoles a ele interligados. 


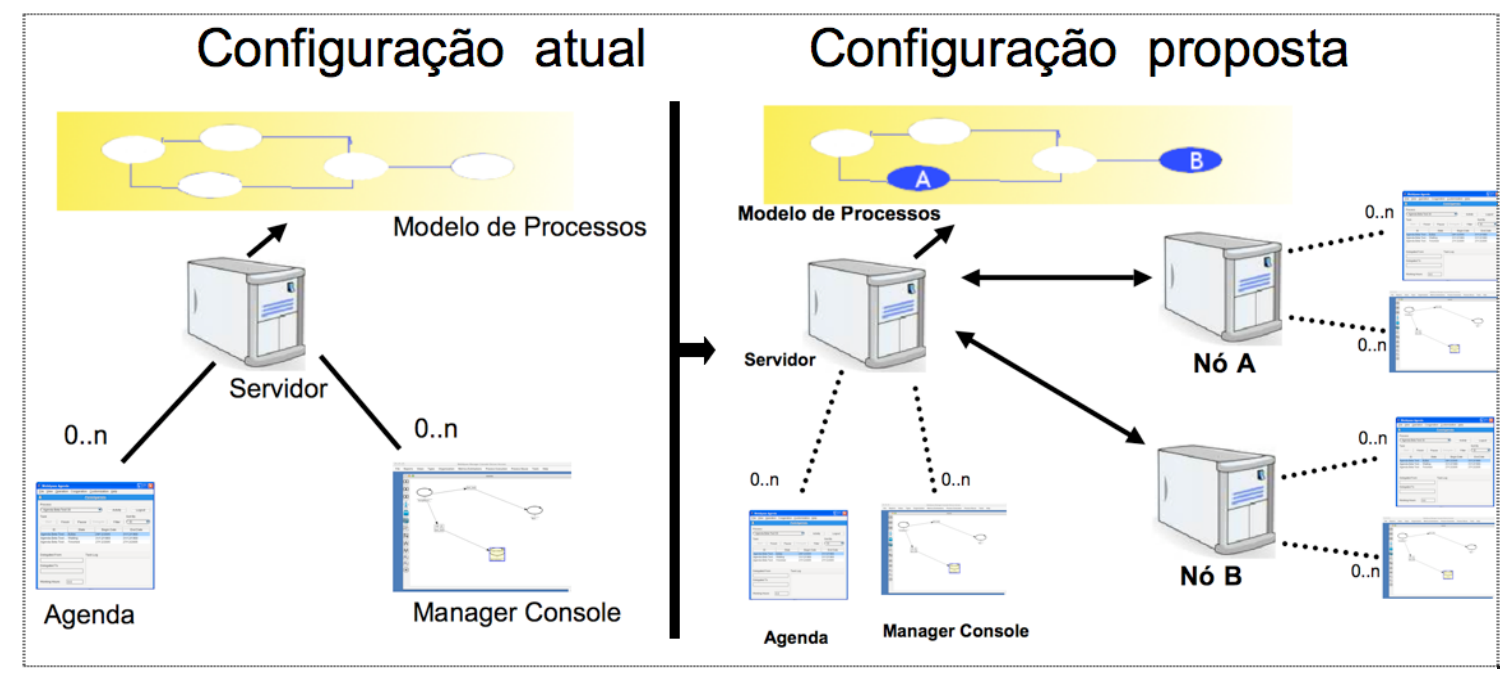

Figura 3 - Escopo do Trabalho

Além das vantagens inerentemente associadas à descentralização de processos, outras vantagens podem ser citadas, tais como: não há ponto único de falha, há aumento da escalabilidade e da concorrência, as informações são armazenadas no domínio da organização representante do site (fornecendo melhores meios para a organização tomar maiores precauções sobre o sigilo dos seus dados).

\subsection{Modelo Arquitetural Proposto}

A arquitetura geral do sistema deve estabelecer uma rede para gerência de dados descentralizados, para que o controle de processos descentralizados possa ser transparente para as camadas já existentes no ambiente, ou seja, novos componentes são adicionados, mas sem modificar a maioria dos componentes já existentes na versão atual do ambiente WebAPSEE.

A Figura 4 destaca, na forma de um diagrama de implantação UML, os principais componentes definidos para atendimento aos requisitos identificados para promover a execução descentralizada de processos ao ambiente WebAPSEE.

O componente de execução é o responsável pela coordenação dos componentes de modelos de processos definidos no ambiente. Estendendo o modelo proposto em [Lima Reis 2003] e descrito em [Lima 2006], este componente promove a coordenação de processos descentralizados, onde além da atualização local de dados, algumas vezes é necessário que eventos sejam propagados e dados sejam trocados entre os sites para sincronia dos mecanismos de execução.

O componente denominado contrato na ilustração da Figura 4 é responsável pelo controle de acesso às informações trocadas entre os sites descentralizados. Estendendo os modelos propostos por [Grundy 1998], [Yan 2006] e [Ritrovato 2002], este componente provê maior flexibilidade no atendimento ao requisito identificado de controle de acesso às informações. Tal flexibilidade é definida pela utilização de um contrato eletrônico que rege as regras de acesso aos dados entre os sites envolvidos nas transações de trocas de processos, permitindo assim a filtragem de informações confidenciais entre os sites envolvidos. 


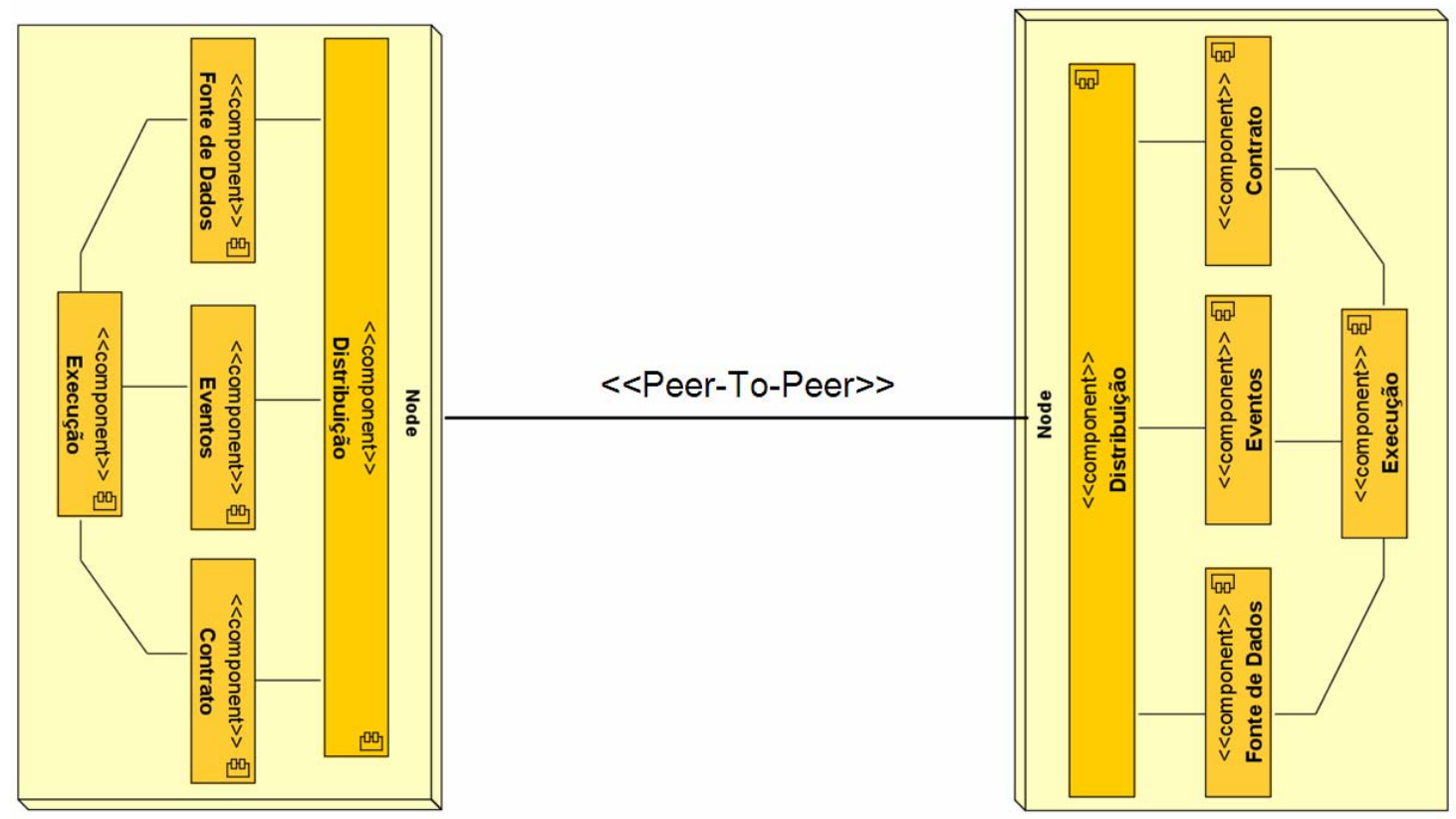

Figura 4 - Arquitetura geral da rede proposta

A definição do contrato eletrônico é proposta como o primeiro passo para o relacionamento automatizado entre clientes e fornecedores, e ilustrado pela Figura 5. Esta abordagem de definição de contratos eletrônicos para reger o acesso às informações é análoga às soluções aplicadas por outros autores em outros contextos, como [Lucena 2005], que aplica o conceito de contratos eletrônicos para reger transações em ambientes multi-agentes.

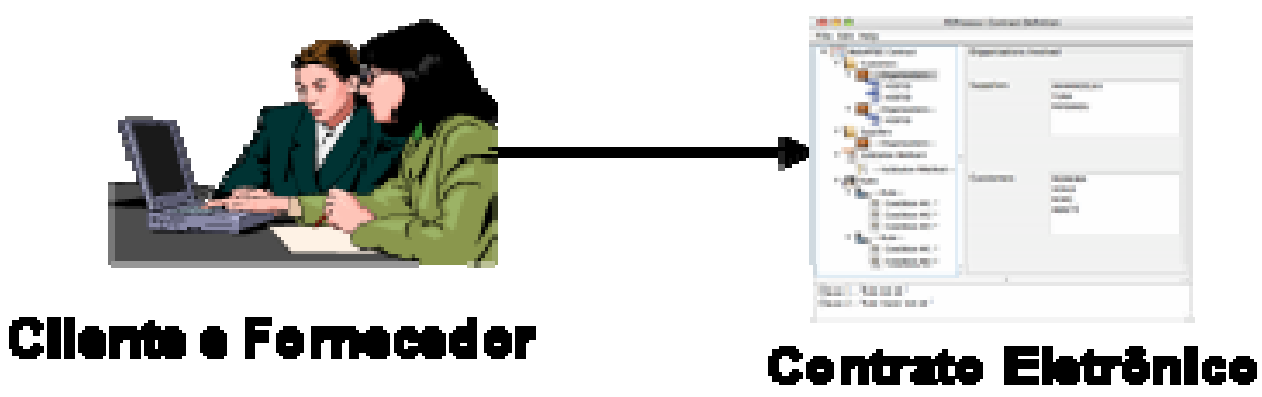

Figura 5 - Definição de Contrato entre Cliente e Fornecedor

A Figura 6 ilustra o processo de delegação de atividades proposto. Estendendo a WebAPSEE-PML atual, foi adicionada a opção de delegação da atividade selecionada para outro fornecedor (site) disponível. Automaticamente o ambiente procura por sites de fornecedores que estejam on-line na rede peer-to-peer para que a transação de delegação de atividade seja concretizada. O protótipo atual da ferramenta já contém os componentes de tratamento das informações do contrato eletrônico, onde há a interpretação das regras e negação ou autorização das operações entre os sites envolvidos. A camada de distribuição peer-to-peer também está implementada, fornecendo a propagação de eventos de sincronização entre os sites. Os esforços de implementação do protótipo se concentram: na implementação dos serviços de transferência de dados a partir da delegação de atividades; na definição das regras de 
consistência que produzirão os eventos de sincronização entre os sites envolvidos na execução descentralizada de processos (assim como regras de consistência de execução e modelagem foram definidas em [Reis 2002] para consistência da execução de processos centralizada no WebAPSE).

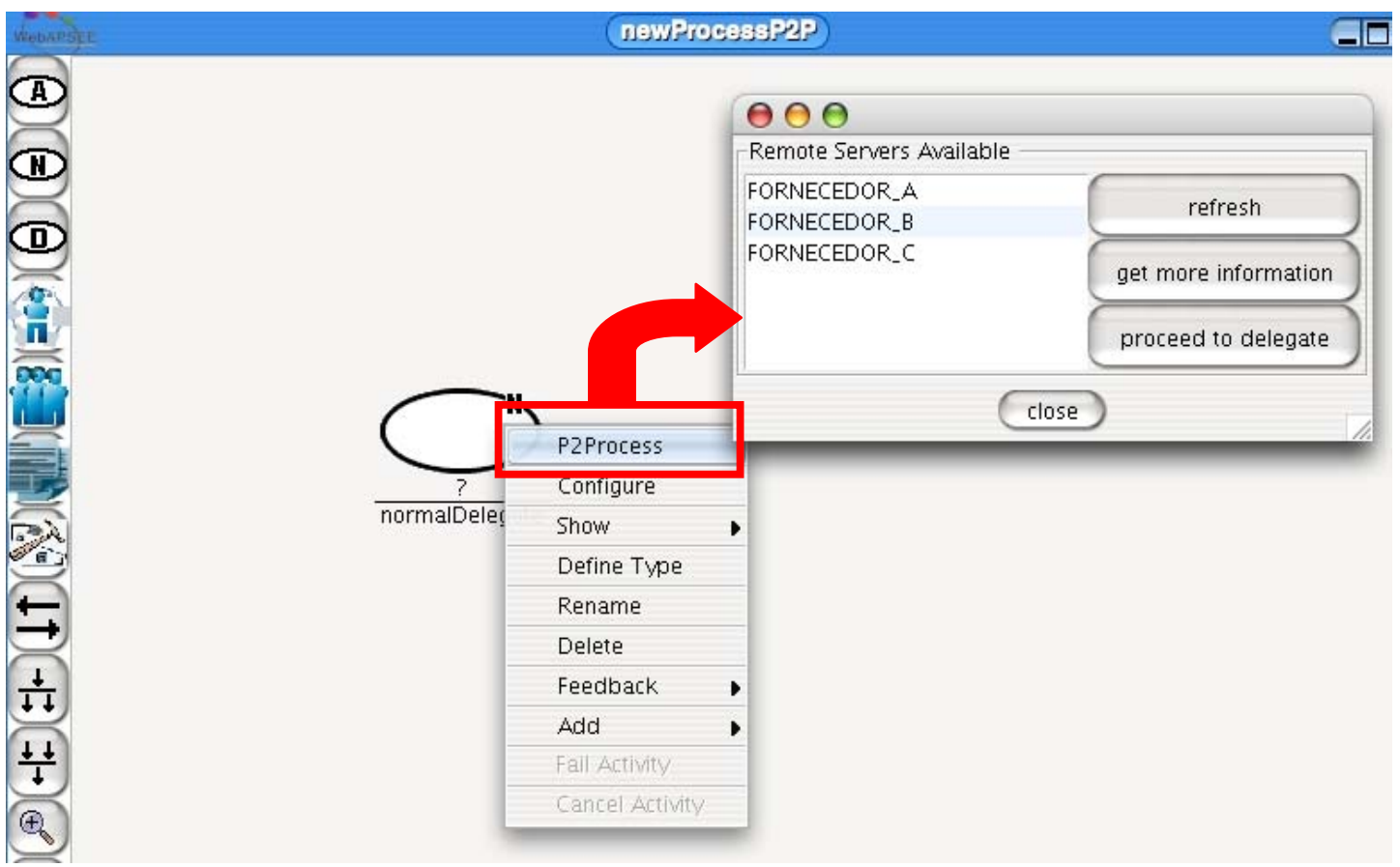

Figura 6 - Delegação de atividades para fornecedor

\subsection{Resultados esperados com o trabalho proposto}

A proposta deste trabalho procura atender alguns aspectos citados pela literatura como dificuldades em projetos de desenvolvimento descentralizado de software.

Assim, a proposta do trabalho se concentra na execução descentralizada de processos, não importando se os sites estejam localizados em diferentes empresas (Outsourcing) ou em filiais da mesma empresa (Insourcing) [Robinson 2004]. Dependendo do contexto organizacional que a ferramenta proposta é aplicada, também é esperada variação na definição da rigidez que o contrato eletrônico definido trata a troca de informações entre os sites envolvidos. A seguir são destacados os principais resultados esperados com a utilização da proposta em projetos reais de desenvolvimento de software.

\subsubsection{Linguagem comum para processos}

É relatada por diversos autores, como [Ebert 2006] e [Sengupta 2006], a necessidade de manutenção de uma linguagem (ou vocabulário) comum entre os membros de equipes dispersas para melhorias na comunicação do andamento das atividades do projeto.

Abordagens como a proposta por [Thiry 2006] permitem o atendimento à definição de processos, mas não atendem à execução automatizada das atividades. Desta forma, a proposta apresentada neste trabalho tem o objetivo de permitir que os benefícios da utilização de uma linguagem de processos comum providos por ferramentas PSEEs em ambientes locais (como o uso do ambiente WebAPSEE [Lima 2006]), sejam também estendidos para projetos descentralizados de desenvolvimento de software. Desta forma a visualização de informações dos projetos descentralizados pode 
ajudar na qualidade de tais projetos, atendendo assim os aspectos citados por [Ebert 2006] e [Sengupta 2006] como essenciais para melhoria da comunicação.

\subsubsection{Sigilo de informações restritas na relação entre as organizações}

O sigilo de informações organizacionais é um aspecto citado por [Wells 2001] e [Sengupta 2006] como imprescindível para real implantação de descentralização de processos. Um aspecto não técnico muito importante envolvido nesta relação de descentralização de processos é confiança entre as partes envolvidas, que determina os dados trocados. Portanto, ferramentas propostas no âmbito da descentralização de processos devem atender este aspecto para ter fatores favoráveis na sua implantação.

Segundo é afirmado por [Bittes 2005], a implantação de melhoria dos processos de uma organização deve levar em consideração aspectos que facilitem a adoção de processos. Desta forma, o atendimento ao sigilo de informações na descentralização de processos pode ajudar a uma melhor implantabilidade de processos, e por conseqüência melhorar a qualidade da coordenação de processos em projetos descentralizados.

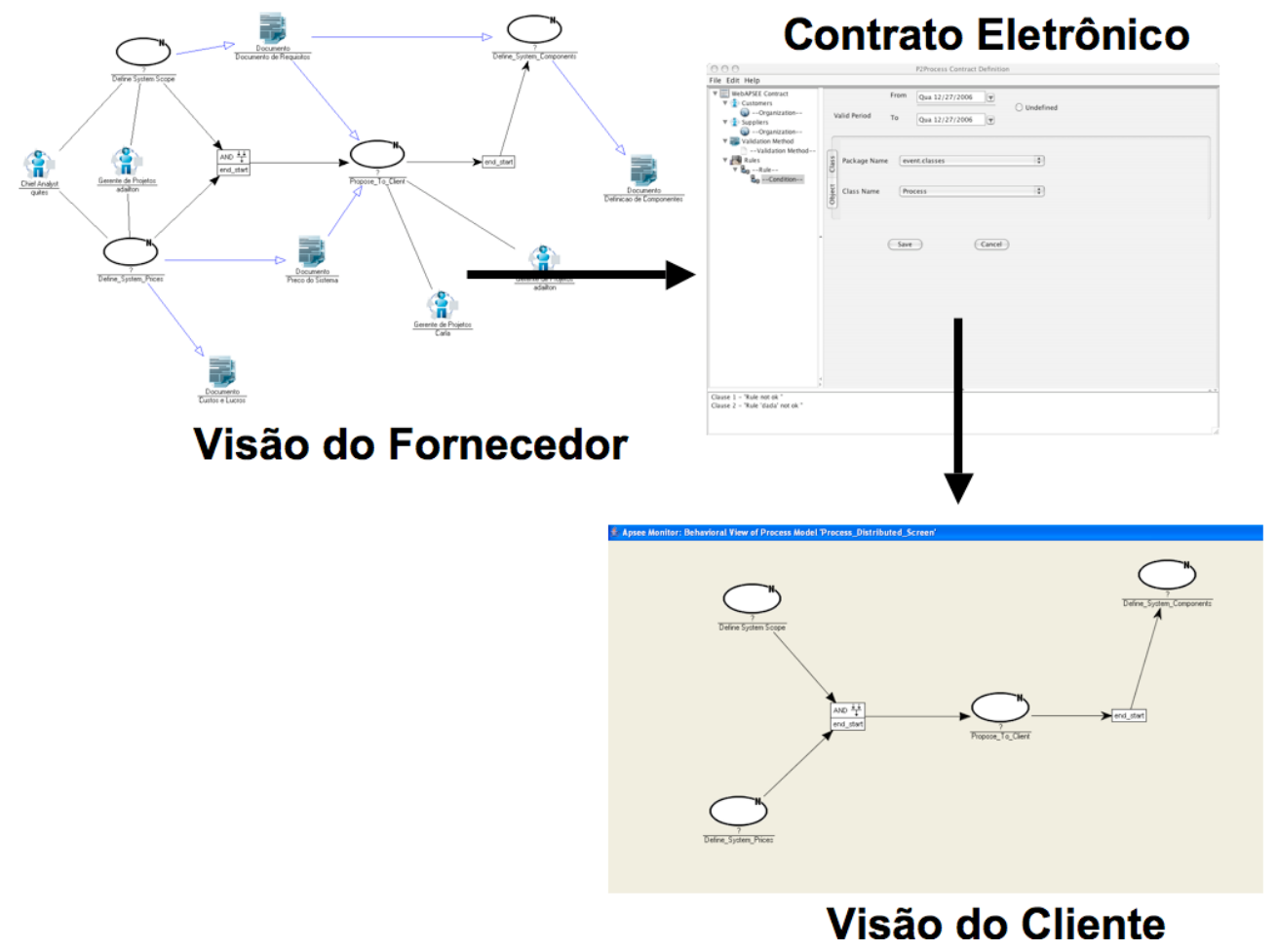

Figura 7 - Resultado restritivo do Contrato de desenvolvimento

A idéia geral da proposta deste trabalho aplica o conceito de contrato eletrônico para definição das regras que irão habilitar ou desabilitar o acesso a dados entre os sites envolvidos na descentralização de processos, conforme ilustrado pela Figura 7. Fornecedores podem configurar a ferramenta através de um contrato eletrônico, permitindo que certas informações dos processos sejam omitidas quando enviadas aos clientes. Desta forma, há o atendimento ao requisito de sigilo de informações entre organizações, que é destacado por [Wells 2001] e [Sengupta 2006]. Em casos que clientes e fornecedores sejam diferentes empresas sem uma relação estrita, espera-se que o contrato eletrônico seja mais restritivo do que no caso em que as empresas envolvidas sejam partes da mesma organização ou mantenham uma relação muito mais estreita. 


\subsubsection{Atendimento às metas do CMMI e MPS.BR}

O trabalho aqui proposto se baseia no raciocínio que modelos de maturidade de processos exigem uma coordenação monitorada entre clientes e fornecedores, e em organizações reais as informações estratégicas devem ser protegidas [Wells 2001] [Sengupta 2006]. O atendimento à meta de coordenação do trabalho com fornecedores de produtos de software é exigido pelo CMMI e MPS.BR. Em casos onde fornecedores externos desenvolvem os produtos de software requisitados, o acompanhamento das atividades do fornecedor é um fator citado como importante para verificação dos métodos e produtos que são desenvolvidos.

A proposta atual de coordenação descentralizada de processos beneficia a monitoração dos processos que estão sendo desenvolvidos pelos fornecedores, permitindo assim maior grau de automação nas tarefas de acompanhamento dos estágios atuais de produção dos fornecedores. Como conseqüência, é esperada facilidade na comunicação entre clientes e fornecedores, que é um dos problemas também identificados pela literatura [Ebert 2006] [Sengupta 2006] [Moitra 2001].

\section{Conclusões e Trabalhos Futuros}

Estendendo requisitos de segurança dos modelos propostos por [Grundy 1998] [Yan 2003] e [Ritrovato 2002], o contrato eletrônico proposto flexibiliza a definição de permissões de acesso entre sites descentralizados. As mensagens de notificação entre sites descentralizados são baseadas na propagação de eventos, de forma similar ao modelo proposto por [Ritrovato 2002] e [Grundy 1998].

O protótipo atual da proposta deste trabalho, que possui a definição de projeto arquitetural baseado na proposta descrita neste trabalho, encontra-se em fase de implementação da sua primeira versão, com a utilização de frameworks peer-to-peer.

Na fase atual de implementação o foco principal do trabalho é a segurança das informações (com contratos eletrônicos) e a arquitetura peer-to-peer para descentralização da execução de processos. Tais características envolvem a coordenação de atividades e troca de artefatos entre os sites envolvidos na distribuição de processos.

A distribuição física dos sites procura representar a distribuição física existente entre diferentes organizações. Como uma possível configuração simplificada da proposta deste trabalho, pode-se ter uma estrutura onde um servidor centralizado armazena todos os dados de todas as organizações envolvidas. Desta forma, o sistema se beneficia apenas da característica de segurança de informações baseada em contrato eletrônico, é avaliado localmente e pode ou não restringir informações baseadas no perfil de usuários (da mesma forma como representado pela Figura 7). Esta configuração pode ser aplicada a casos específicos, onde a escalabilidade seja menos importante que a facilidade de implantação do sistema em um único site.

Um dos aspectos citados por [Ebert 2006] é a dificuldade de escolher quais são os parceiros em potencial que são mais capazes de atender certa demanda de desenvolvimento. Modelos de maturidade de processos, como o CMMI [CMMI 2007] e o MPS.BR [Weber 2006] definem que o processo de escolha de fornecedores de TI deve ser documentado e baseado em critérios bem definidos. Assim, como trabalho futuro, a característica de reputação em redes peer-to-peer [Kinateder 2003] pode ser considerada auxiliar na coleta de métricas para escolha de possíveis fornecedores de software a partir do histórico [Ebert 2006]. Segundo [Kinateder 2003], a reputação de 
sistemas é aplicada em sistemas computacionais onde negócios estão envolvidos. No domínio específico do problema tratado neste trabalho (execução descentralizada de processos de software), podem ser monitorados diversos aspectos, podendo citar: tempo médio em que o host $\mathrm{X}$ fica indisponível na rede peer-to-peer; tempo médio nos quais artefatos do tipo Y são entregues; número médio de tarefas que são delegadas e não cumpridas pelo host Z; dentre outros.

\section{Referências}

Beanshaul, I.Z.; Kaiser, G.E. (1998) "Federating Process-Centered Environments: The OZ Experience”. In: Automated Software Engineering 5.

Bittes, José Márcio; Santoro, Flávia Maria; Borges, Marcos R. S. (2005) “A Implantabilidade como Atributo de Qualidade de Software”. In: SBQS - Simpósio Brasileiro de Qualidade de Software (SBQS’06), Porto Alegre, Brazil.

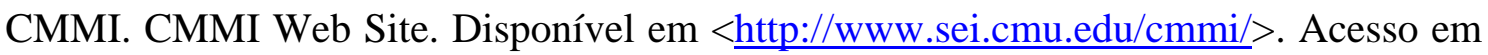
18 de janeiro de 2007.

Conradi, Reidar; et al. (1994) “EPOS: Object Oriented Cooperative Process Modeling”. In: Software Process Modeling and Technology, Research Studies Press LTD.

Derniame, Jean-Claude, et al. (2002) “A Comparative Review of Process-Centered Software Engineering Environments”. In: Annals of Software Engineering 14, p. 311-340.

Ebert, Christopher. (2006) “Global Software Engineering”. IEEE ReadyNote (e-Book), IEEE Computer Society, Los Alamitos, USA.

Feldmann, Raimund L.; Rombach, Dieter; Kobitzsch, W. (2001) “Outsourcing In India”. IEEE Software, 18(2):78-86, Março/Abril.

Fuggetta, Alfonso. (2000) "Software Process: A Roadmap”. In Proc. of The Future of Software Engineering, ICSE’2000, Limerick, Ireland.

Fuggetta, Alfonso; et al. (2000) "Support for Software Federations: the PIE Platform”. European Workshop on Software Process Technology.

Gimenes, Itana M. S. (1994) "Uma Introdução ao Processo de Engenharia de Software: ambientes e formalismos”. Trabalho apresentado na 13. Jornada de Atualização em Informática, Caxambu.

Grundy, J.C., et al. (1998) "An architecture and environment for decentralized, internetwide software process modeling and enactment”. In: IEEE Internet Computing: Special Issue on Software Engineering via the Internet, Vol. 2, No. 5, IEEE CS Press, September/October.

Herbsleb, James D.; et al. (2001) "An Empirical Study of Global Software Development: Distance and Speed”. In: Proceedings International Software Engineering 2001, IEEE CS Press, Los Alamitos, Califórnia.

Kinateder, Michael; Rothermel, Kurt. (2004) “Architecture and Algorithms for a Distributed Reputation System”. Springer-Verlag, Berlin, Heidelberg.

Lima, Adailton Magalhães; Reis, Rodrigo Q.; Lima Reis, Carla A. (2006) "Gerência Flexível de Processos de Software com o Ambiente WebAPSEE”. 19. Simpósio Brasileiro de Engenharia de Software - Sessão de Ferramentas, Outubro. 
Lucena, Carlos J. P.; Silva, Viviane. (2005) "Classifying and Describing Agent Contracts and Norms”. In: Agents, Norms and Institutions for Regulated Multiagent Systems - ANIREM, Utrecht, Holanda, Julho.

Moitra, Deependra; Herbsleb, James D. (2001) “Global Software Development”. In proceedings, International Conference on Software Engineering (ICSE 2001) Toronto, Canada, May 15-18, pp. 81-90.

Montoni, M. et al. (2006) "Uma Abordagem de Garantia de Qualidade de Processos e Produtos de Software com Apoio de Gerência de Conhecimento na Estação TABA”. V Simpósio Brasileiro de Qualidade de Software (SBQS'06). Vila Velha, Brasil.

Reis, Carla A. Lima. (2003) "Uma Abordagem Flexível para Execução de Processos de Software Evolutivos", Tese de Doutorado, Instituto de Informática, Universidade Federal do Rio Grande do Sul.

Reis, Rodrigo Quites; Lima Reis, C.A.; Nunes, D.J. (2002) “APSEE-StaticPolicy: Automatic Verification of Static Policies on Software Process Models. Accepted for publication in: Annals of Software Engineering, Volume 14 on Process-Based Software Engineering. Kluwer. Outubro.

Reis, Rodrigo Quites. (2002) “APSEE-Reuse: um Meta-Modelo para Apoiar a Reutilização de Processos de Software”. Tese (Doutorado em Ciência da Computação) - Instituto de Informática, UFRGS, Porto Alegre.

Ritrovato, Pierluigi; Gaeta, Matteo. (2002) "Generalised Environment for Process Management in Cooperative Software Engineering”. In: Proceedings. 26th Annual International Computer Software and Applications Conference.

Robinson, M.; Kalakota, R. (2004) "Offshore Outsourcing: Business Models, ROI and Best Practices”. EUA: Mivar Press.

Sengupta, Bikram; Chandra, Satish; Sinhá, Vibha. (2006) “A Research Agenda for Distributed Software Development”. In: Proceedings of International Conference on Software Engineering (ICSE’06), Shanghai, China.

Taylor, Richard N.; Bolcer, Gregory A. (1996) "Endeavors: A Process System Integration Infrastructure”. In: Proceedings of International Conference on Software Process (ICSP 4), Brighton, Reino Unido.

Yan, Jun; YANG, Yun; Raikundalia, Gitesh K. (2003) "Decentralised Coordination for Software Process Enactment”. In: Lecture Notes in Computer Science, Heidelberg: Springer Berlin.

Weber, Kival; et al. (2006) "Melhoria de Processo do Software Brasileiro (MPS.BR): um programa Mobilizador”. In: $32^{\mathrm{a}}$ Conferência Latino-Americana de Informática (CLEI 2006), Santiago, Chile, Agosto.

Wells, Thomas O.; Braunfeld, Roger. (2001) "Protecting Your Most Valuable Asset: Intellectual Property”. In: IEEE Special Issue in IT Professional, Volume: 3, Issue: 2, pag. 11-17, Marco-Abril.

Thiry, Marcello et al. (2006) "Uma Abordagem para a Modelagem Colaborativa de Processos de Software em Micro e Pequenas Empresas”. V Simpósio Brasileiro de Qualidade de Software (SBQS'06). Vila Velha, Brasil. 